\title{
SERVICES OF COMPUTATIONAL NEUROBIOLOGY TASKS, BASED ON THE DISTRIBUTED MODULAR PLATFORM «DIGITAL LABORATORY» NRC «KURCHATOV INSTITUTE»
}

\author{
I.M. Enyagina a A.N. Polyakov, D.S. Kokovin \\ NRC “Kurchatov Institute”, 1 Akademika Kurchatova sq., Moscow, 123182, Russia \\ E-mail: aEnyagina_IM@nrcki.ru
}

This paper describes program services for performing tasks of computational neurobiology, based on the distributed modular platform "Digital Laboratory" NRC «Kurchatov Institute». These services expanded the system "Neuroimaging" for storing, processing and analyzing experimental MRI / fMRI data of the human brain. The main goal of creating these program services is the software implementation of methods for calculating the functional connectivity of regions of the human brain at rest using fMRI data, and visualization of the results.

Keywords: analysis of experimental data, IT system, MRI, fMRI, neuroimaging, Digital Laboratory. computational neurobiology.

Irina Enyagina, Andrey Polyakov, Dmitry Kokovin 


\section{Introduction}

One of the most actual tasks of neurobiology today is the study of the anatomical structure and functional neuronal activity of the human brain. Results of such studies are used in clinical practice and research activities: machine learning, brain-computer interface, analysis of the consequences of proton therapy in brain tumors, the effect of radiation on human cognitive abilities, emergency recognition of the type of stroke, the early diagnosis of Alzheimer's disease, and much more. The most popular in this area are the methods of MRI and fMRI, based on the principle of nuclear magnetic resonance. These are new and actively developing methods. The technologies for conducting MRI / fMRI experiments and analyzing the obtained experimental data are constantly being improved. As a result, there is a need for the constant development of IT systems for working with experimental data from MRI / fMRI. At the Kurchatov Institute MRI/fMRI experiments are performed on the MRI scanner (tomograph) of the Resource Center «Cognimed». For storage, processing and analysis of the obtained MRI/ fMRI experimental data on the basis of the distributed-modular platform "Digital Laboratory", the system "Neuroimaging" was created, with the involvement of the supercomputer of the NRC KI. This system provides the ability to process data using standard specialized packages and also by unique author's methods. This article presents two new software services of the "Neuroimaging" system. The first service "MFS Method" "performs processing and analysis of fMRI data of the human brain at rest using a unique functional segmentation method developed by NRC KI researchers. The second service, «Visualization BrainBrowser», allows to visualize the results of fMRI data processing.

\section{Materials and methods}

\section{1 «Digital Laboratory» Platform}

Information-analytical platform "Digital Laboratory" [1],[2] is a distributed modular system for intensive work with data and metadata in a heterogeneous data warehouse, with the function of dynamically changing by the user both the data itself and the metadata models [3],[4],[5]. Dynamic modification of data models and types of links between them allows you to develop the functionality of the system and develop new methods of data processing and analysis. The Digital Laboratory platform acts as a data flow manager for organizing the transformation and transfer of data between individual elements of the information environment as a data processing and analysis system. The key component of the Digital Lab platform is the metadata repository, the main elements of which are data and metadata models. The interface to these models has a standardized format and is provided by services [1]. On the basis of the Digital Laboratory platform, it is possible to create both simple modules for processing and analyzing homogeneous data, and complex modules for processing and analyzing heterogeneous data [2].

\subsection{System «Neuroimaging»}

The MRI and fMRI methods has opened up new opportunities for studying the anatomical structure and functional neuronal activity of the human brain. However, as the number of MRI/fMRI experiments increased, the problem arose of fast processing and analysis of the flows of experimental MRI / fMRI data, and their ordered centralized storage. For this goal, on the base of the "Digital Laboratory» platform, the System "Neuroimaging" [6] was developed and implemented, for storing, analyzing and visualizing experimental MRI/fMRI data, with use of the supercomputer NRC "Kurchatov Institute" as a computing resource. System allows to conduct scientific research in the field of studying the anatomical structure and functional neuronal architecture of the human brain, using traditional methods and tools of mathematical modeling, and unique techniques developed by researchers NRC KI. The System "Neuroimaging" allows many times to speed up, simplify and expand the possibilities of processing and analysis of MRI and fMRI data due to the parallelization of computational processes at the supercomputer nodes, providing a wide range of specialized processing and analysis tools, with access from anywhere at any time by web interface. 


\section{Results}

\subsection{Program service «Method MFS»}

Functional segmentation method (MFS) is applied for allocation of functionally homogeneous regions of the human brain at rest [7],[8]. It is based on the correlation approach and as a measure of similarity uses Pearson's correlation, and also allows you to select regions whose voxels have a high level of correlation. In this case, a voxel means a set of neighboring neurons within a cubic space a given size (from 1 to $3 \mathrm{~mm}$, depending on the type of experiment being carried out).

The main goal of creating the program service "Method MFS" was to create a tool for automating the work of users with a program that implements the MFS method [fig. 1]. At the same time, it was necessary to develop the most simple procedure for user interaction with the program, which does not require special IT skills. It was also necessary to organize remote access and create the ability to visualize the results.

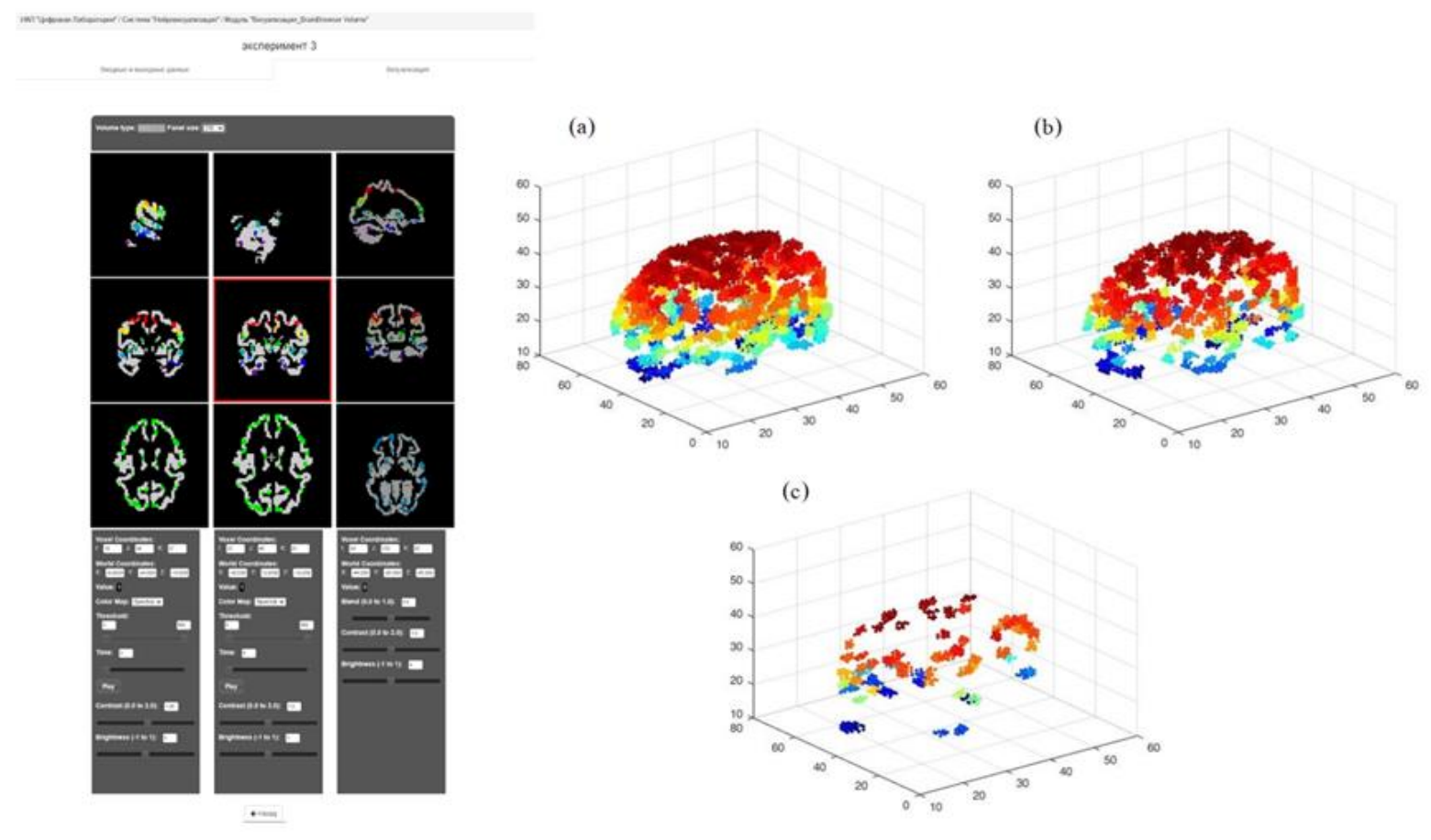

Figure 1. Results of applying the MFS method to fMRI data

An algorithm was designed that implements the workflow of data within the framework of the functioning of the service "MFS Method", then a computer model was developed [8], consisting of the following components: User's PCs. External personal computers that users use to work with the "Method MFS" module; User web interface. A software shell that automates the procedures for starting a task and obtaining results, as well as providing the ability to remotely access the system; Data flow control unit. Internal software module of the IAP "Digital Laboratory", coordinating the exchange of data streams between the components of the model (user interface, data separation unit, data warehouse, virtual machine); Block for separating input data. Separation of metadata from data; Data storage. Data storage server of the "Digital Laboratory", which provides centralized orderly storage of input data, clean input data, metadata, output data; Computing element. A virtual machine on which a program is deployed that applies the MFS to input data. This computer model was implemented in the form of the program service «Method MFC» of the System "Neuroimaging". 


\subsection{Program service «Visualization BrainBrowsen»}

Program service «Visualization BrainBrowser» allows to visualize 3D models of the anatomical structure and functional activity of the human brain, obtained as a result of processing and analysis of experimental MRI / fMRI data [fig. 2]. The module uses the capabilities of the freely distributed specialized visual editor BrainBrowser for viewing surfaces and volumetric structures of the brain. BrainBrowser lets to explore any volume of a MINC file or 3D object (such as surfaces from CIVET, Freesurfer, or Wavefront objects). This step allows you to conveniently perform quality control, which is often critical before moving on to further analysis or transferring large amounts of data, especially if data format conversion steps have been applied.

The module consists of two sections: Surface. This section provides system users with access to the BrainBrowser Surface Viewer v2.5.2 software package, which allows visualization of the brain surface based on the results of analysis and processing of MRI / fMRI data uploaded by the user; Volume. This section provides system users with access to the BrainBrowser Volume Viewer v2.5.2 software package, which allows visualization of the volumetric structures of the brain based on the results of analysis and processing of MRI / fMRI data uploaded by the user.

The following free software was used: Java (version 8 and higher), JavaScript, HTML 5; formats for describing metadata XML; DBMS MySQL version 5.5; J2EE interfaces; frameworks Spring, Hibernate, Activiti; Apache web server and Tomcat 8.5 application server.

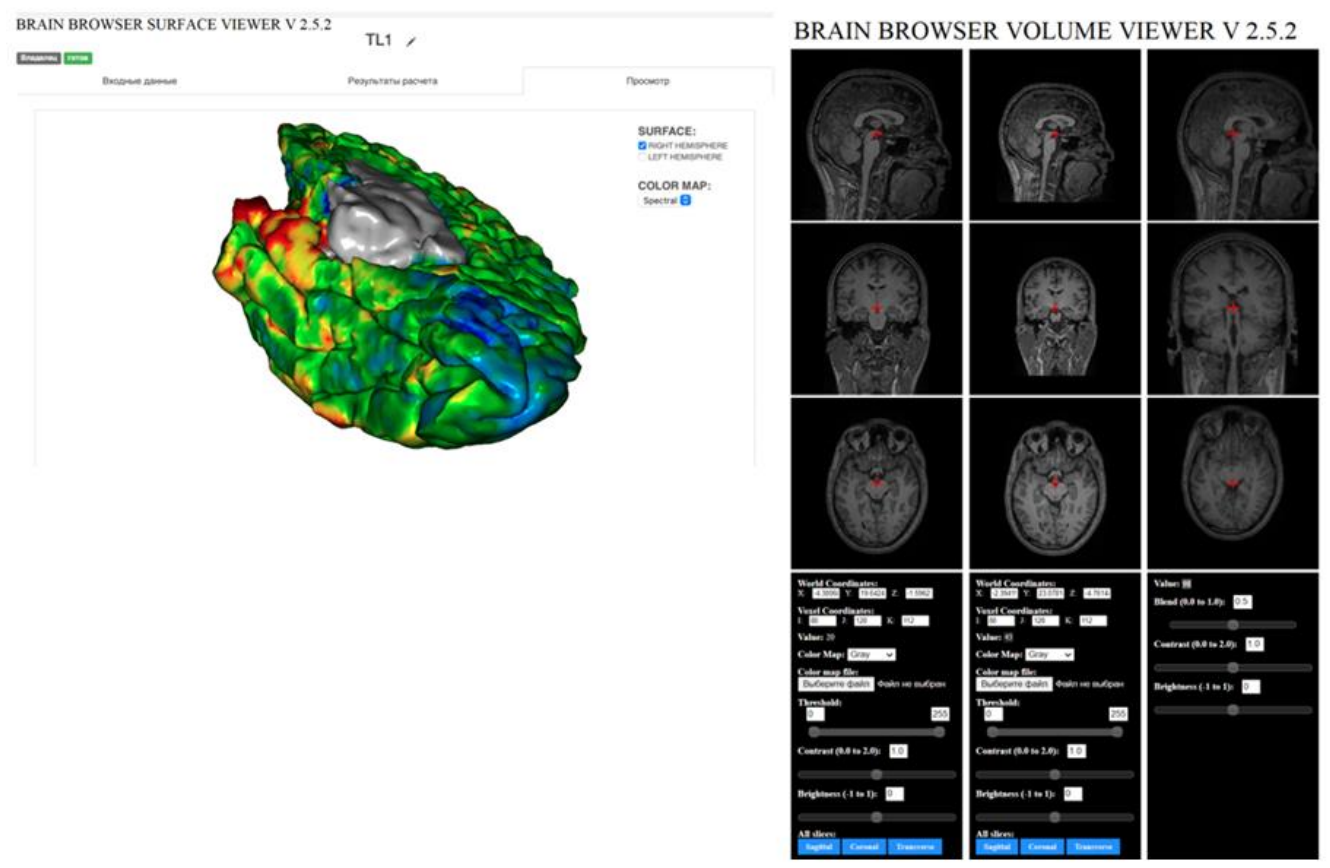

Figure 2. BrainBrowser: visualization of the results of processing MRI/fMRI data

\section{Conclusion}

We have presented two new program services of the System "Neuroimaging" for working with experimental MRI / fMRI data of the human brain. This system was developed based on the "Digital Laboratory" Platform NRC KI. The " Module MFS" service is a software implementation of the author's method of functional segmentation, developed by the NRC KI researchers. The service provides an opportunity to apply the MFS method to fMRI data at rest. The "Visualization BrainBrowser" service provides an opportunity to visualize the results of processing MRI / fMRI data in two modes: surface or volume. The creation of these services provides new opportunities for researchers to solve neurobiological tasks in the field of studying the anatomical structure and functional neuronal architecture of the human brain. 


\section{Acknowledgement}

This works was supported by the Kurchatov Institute research activities on the project "Creation of a distributed modular research and development platform "Digital Laboratory"» approved by order of the Kurchatov Institute on July 02, 2020, No. 1055 and by the RFBR research project No 18-29-23020 mk.

\section{References}

[1] A. N. Polyakov et al., “'Digital Lab' platform as an environment for scientific research and development at the Kurchatov Institute," Tr. Inst. Sist. Program. RAN, 32 (5), 143-152 (2020).

[2] Polyakov A., Kokovin D., Poyda A., Zhizhin M., Andreev A., Govorov A., Ilyin V. Toolkit for intensive work with metadata in specialized information systems. Procedia Computer Science, vol. 119, 2017, pp. 59-64.

[3] Bubenko Janis A., jr. From Information Algebra to Enterprise Modelling and Ontologies - a Historical Perspective on Modelling for Information Systems. In Conceptual Modelling in Information Systems Engineering, Springer, 2007, pp. 1-18.

[4] Burgess N. Scaling an RNS number using the core function. In Proc. of the 16th IEEE Symposium on Computer Arithmetic, 2003. pp. 262-269.

[5] Jenn Riley. Understanding Metadata: What is Metadata, and What is it For? National Information Standards Organization, 2017, 49 p.

[6] Enyagina I.M., Polyakov A.N., Poyda A.A., Ushakov V.L. System for Automatic Processing and Analysis of MRI/fMRI Data on the Kurchatov Institute Supercomputer. EPJ Web of Conferences, vol. 226, 2020, article no. 03006 .

[7] S. Kozlov, A. Poyda, V. Orlov, V. Malakhov, V. Ushakov, and M. Sharaev, "Selection of functionally homogeneous brain regions based on correlation-clustering analysis," Proc. Comput. Sci. $169,519-526(2020)$.

[8] I.M. Enyagina et al., "Implementing Methods for Calculating the Functional Connectivity of Regions of the Human Brain at Rest and Neuroimaging Using Data of Functional Nuclear Magnetic Resonance Imaging (fMRI)», ISSN 1547-4771, Physics of Particles and Nuclei Letters, 2021, Vol. 18, No. 4, pp. 496-501. (C) Pleiades Publishing, Ltd., 2021. 